\title{
Quarter Wavelength Matching in Dielectric Resonator Bandpass Filter
}

\author{
M. F. Ain, Z. A. Ahmad, M.A. Othman, I. A. Zubir, \\ S. D. Hutagalung. \\ School of Electrical and Electronics / Material and Mineral \\ Resources Engineering, USM, 14300 Penang, Malaysia. \\ e-mail: mohdfadzilain@yahoo.com.my
}

\author{
A. A. Sulaiman, A. Othman \\ Faculty of Electrical Engineering, \\ UiTM, 40450 Shah Alam, Malaysia \\ e-mail: asari100@yahoo.com
}

\begin{abstract}
This paper focuses on the analysis to find suitable locations to place dielectric resonators on microstrip transmission line in order to obtain a high coupling effect. A novel design of an $\mathrm{X}$-Band bandpass filter from a combination of a simple transmission line and three cylindrical dielectric resonators are used in this analysis. The three dielectric resonators are same in permittivity and radius of 60 and $2.55 \mathrm{~mm}$, respectively. This analysis found that the coupling effect can be maximized by placing dielectric resonators a distance of quarter or half wavelength from each other.
\end{abstract}

Keywords- Bandpass filter; Dielectric resonator; wideband.

\section{INTRODUCTION}

Dielectric resonator (DR) offers a lot of advantages in increasing the performance of RF and microwave devices which make it as a unique feature in some of applications where size and Q-factor are very important. It is an ideal candidate for wireless application for a low design profile and wideband applications [1-2]. However, the performances of the most distributed resonators are limited due to the use of effective dielectric constant and discontinuity of the transmission line. Strip line structures have grasped substantial research interests due to the advantages such as ease in the circuit realization in both series and shunt stubs and no need for via holes [3-5]. The design procedure of bandpass filter microstrip lines is well documented in literature [6].

The advantages of dielectric resonators are more compact, higher temperature stability and easy to be practically applied in microwave circuits. Moreover they are amenable in multitechnology such as printed circuit and surface mounts technology. The dielectric resonators are also usually shielded to prevent radiation as well as maintain a high-Q that required by filter and oscillator circuits [7].

Microstrip bandpass filters play important roles in microwave circuits and systems. The most famous filter in microwave applications is the bandpass filter [3]. The filter can be narrow- or wide-bands depend on the applications. A narrow-band bandpass device is designed for stringent specifications of passband insertion loss and stopband rejection. While a wide-band bandpass filter is normally used for high data transmission involving a lot of video and data communications [6]. Their practical realization varies depending on the applications.

The DR filters are good for mobile and satellite communications. A typical DR filter consists of a number of dielectric resonators that are mounted in a planar configuration to obtain a good resonant frequency [7]. The relative dielectric constant of the material for constructing DR in microwave filter generally is chosen from a higher value compared to the base substrate. The primary advantage in using a high dielectric constant is to miniaturize the filter size. The size of DR filter is considerably smaller compared to the dimension of waveguide filters operating at the same frequency. Furthermore, these DR filters are employed to replace waveguide filters in applications such as satellite communication systems where the planar filters cannot be used because of their inherently high loss. The challenges in designing a DR bandpass filter is how to choose a correct location to place the ceramic when few numbers of dielectric resonators are applied in the design.

In this paper, a detail analysis on the placement of the dielectric resonators in a new structure of a bandpass filter has been done in order to show the affect of the location on the matching of the filter. The optimum coupling effect in the filter was obtained from the matching position of the resonators on the microstrip line. The circuit was simulated with the help of CST Microwave Studio.

\section{DESIGN METHODOLOGY}

The size, location and shape of the dielectric affect the impedance matching of a microwave circuit. In this project, three dielectric resonators were excited with a simple microstrip line in order to obtain the optimum coupling effect. A match combination of dielectric resonators and microwave circuit capable to generate an additional coupling effect that can be merged together to produce a wideband device as well as increasing the transmitting power and reduce the insertion loss. This combination proficiently produces a low design profile.

There is an inversely proportional between size and dielectric constant. A high dielectric constant is required to reduce circuit size of a device. A significant miniaturization can be achieved, thus high-quality filters can be realized. 